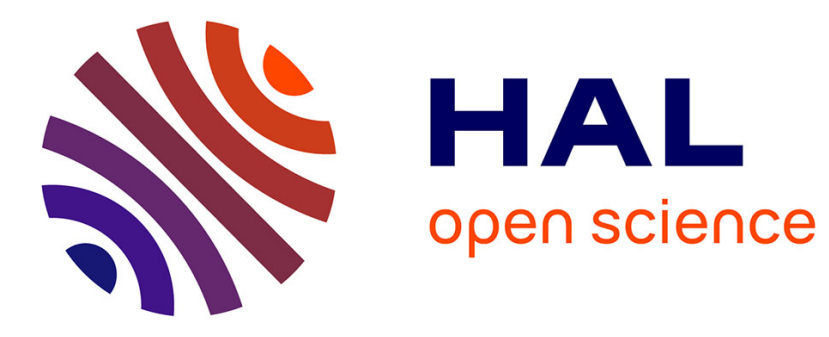

\title{
Excited State Pathways Leading to Formation of Adenine Dimers
}

Akos Banyasz, Lara Martinez-Fernandez, Tiia-Maaria Ketola, A. Munoz-Losa, Luciana Esposito, Dimitra Markovitsi, Roberto Improta

\section{To cite this version:}

Akos Banyasz, Lara Martinez-Fernandez, Tiia-Maaria Ketola, A. Munoz-Losa, Luciana Esposito, et al.. Excited State Pathways Leading to Formation of Adenine Dimers. Journal of Physical Chemistry Letters, 2016, 7, pp.2020-2023. 10.1021/acs.jpclett.6b00660 . cea-01360050

\section{HAL Id: cea-01360050 https://hal-cea.archives-ouvertes.fr/cea-01360050}

Submitted on 5 Sep 2016

HAL is a multi-disciplinary open access archive for the deposit and dissemination of scientific research documents, whether they are published or not. The documents may come from teaching and research institutions in France or abroad, or from public or private research centers.
L'archive ouverte pluridisciplinaire HAL, est destinée au dépôt et à la diffusion de documents scientifiques de niveau recherche, publiés ou non, émanant des établissements d'enseignement et de recherche français ou étrangers, des laboratoires publics ou privés. 


\section{Excited State Pathways Leading to Formation of}

\section{Adenine Dimers}

Akos Banyasz, ${ }^{a}$ Lara Martinez-Fernandez, ${ }^{b}$ Tiia-Maaria Ketola, ${ }^{a ¥}$ Aurora Muñoz-Losa,${ }^{a+}$

Luciana Esposito, ${ }^{b}$ Dimitra Markovitsi ${ }^{* a}$ and Roberto Improta $*^{b}$

${ }^{a}$ LIDYL, CEA, CNRS, Université Paris-Saclay, F-91191 Gif-sur-Yvette, France

${ }^{\mathrm{b}}$ Istituto Biostrutture e Bioimmagini- Consiglio Nazionale delle Ricerche, Via Mezzocannone 16, I-80134 Napoli, Italy

\section{AUTHOR INFORMATION}

\section{Corresponding Authors}

dimitra.markovitsi@cea.fr

robimp@unina.it

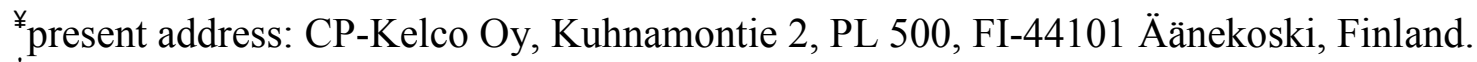

$\dagger$ present address: Institute of Theoretical Chemistry, University of Vienna, Währinger Str. 17, 1090 Vienna, Austria. 


\section{ABSTRACT}

The reaction intermediate in the path leading to $\mathrm{UV}$-induced formation of adenine dimers $\mathrm{A}=\mathrm{A}$ and $\mathrm{AA}^{*}$ is identified for the first time quantum mechanically, using PCM/TD-DFT calculations on $(\mathrm{dA})_{2}$. In parallel, its fingerprint is detected in the absorption spectra recorded on the millisecond time-scale for the single strand $(\mathrm{dA})_{20}$.

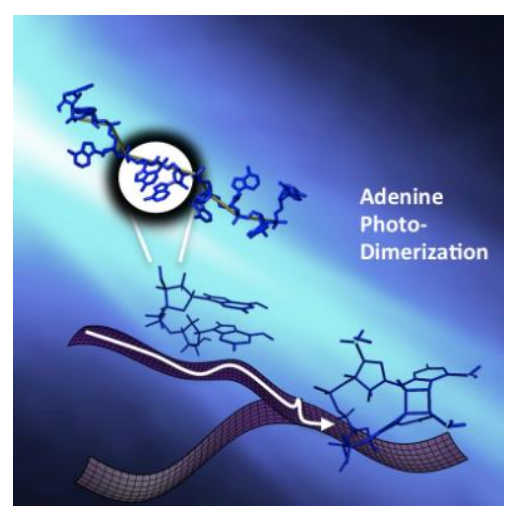


The characterization of photochemical reactions of nucleic acids is important in connection with the UV-induced damage to the genetic code. Although a large number of publications deal with pyrimidine dimerization, very few focus on adenine dimer formation. ${ }^{1-8}$ Yet, the photodimerization quantum yield of adenine single strands is the order of $10^{-3}, 6$ comparable to that of (6-4)pyrimidine-pyrimidone adducts (64PPs) in thymine single strands, ${ }^{9}$ which have received considerable attention. ${ }^{10}$ Intriguingly, the photodimerization yields dramatically drop in DNA duplexes and in RNA strands., ${ }^{3,}$, ${ }^{11}$ Experimental studies demonstrated that irradiation around $260 \mathrm{~nm}$ leads to the formation of two different types of adenine dimers AA* and A=A (Fig. 1) and suggested that they have a common azetidine intermediate. ${ }^{5}$ Here, we report the first theoretical study exploring the excited state pathways that lead to the azetidine precursor. The fingerprint of the reaction intermediate, which, according to our calculations, presents weak electronic transitions at longer wavelengths compared to $\mathrm{AA}^{*}$ and $\mathrm{A}=\mathrm{A}$, is detected in the absorption spectra recorded at $20 \mathrm{~ms}$ following $266 \mathrm{~nm}$ excitation by $5 \mathrm{~ns}$ laser pulses of the single strand $(\mathrm{dA})_{20}$.

Using extensive molecular dynamics (MD) analysis of (dA) $)_{5}$ (see SI, Fig. SI-1), we found that a significant percentage of adenines undergoes syn/anti conformational equilibrium, syn glycosidic conformers being particularly stable for the 5'-terminal base. Then, the four (AA) dinucleotides corresponding to the different combinations of conformers, namely (AA) $)_{a n t i-a n t i},(\mathrm{AA})_{s y n-a n t i},(\mathrm{AA})_{a n t i-s y n}$, and (AA) $)_{\text {syn-syn, }}$, have been optimized at the M052X/6-31G(d) level, ${ }^{12}$ including bulk solvent effects by means of the Polarizable Continuum Model (PCM). ${ }^{13} \mathrm{We}$ have focused on $\mathrm{C} 2$ '-endo/C2'-endo conformers, since MD simulations predict that most of the 'inner bases' (those more representative of the 
behavior in long strands) adopts a $\mathrm{C}^{2}$ '-endo puckering. At the PCM/M052X level of theory, the most stable conformer is (AA) syn-anti $($ Fig. SI-3 and Table SI-1), which is stabilized by a strong hydrogen bond between the terminal $\mathrm{CH}_{2} \mathrm{OH}$ group and $\mathrm{N} 3$ atom of the 5'-terminal adenine $\left({ }^{5} \mathrm{~N} 3\right)$ (Fig. 1). When the molecules of the first solvation shell (see SI, Fig. SI-2) are included, (AA) anti-anti $i$ found to be the most stable conformer, but the difference in energy with respect to $(\mathrm{AA})_{\text {syn-anti }}$ is only $4.0 \mathrm{kcal} / \mathrm{mol}$, suggesting that a very small, but non-zero, percentage of the AA steps adopts this conformation.
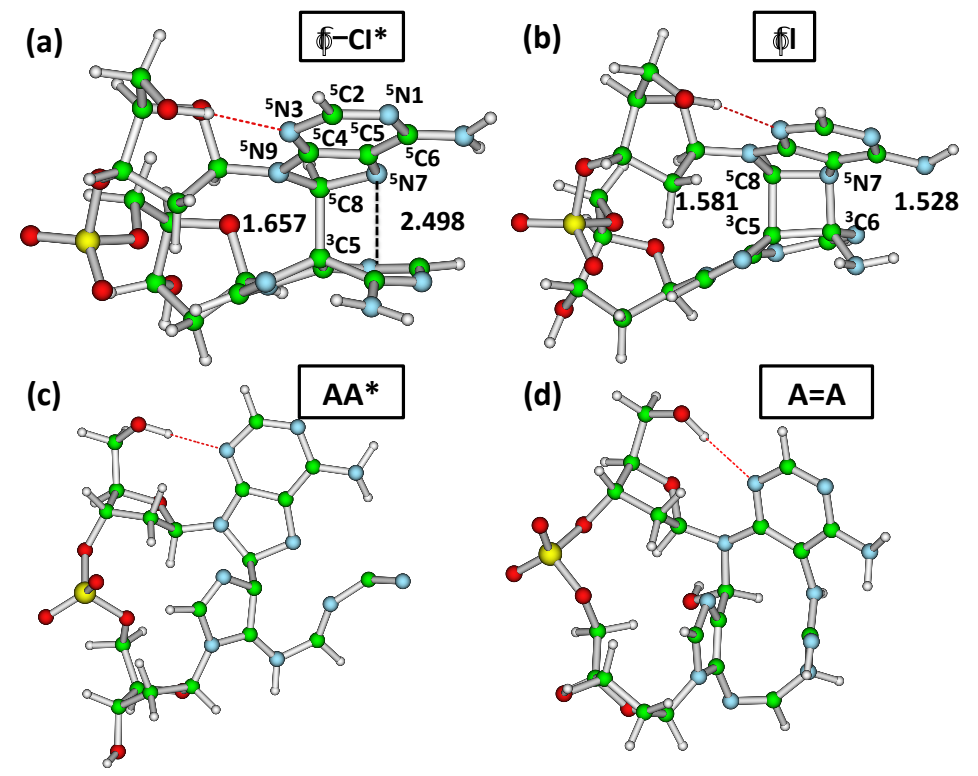

Figure 1. Schematic drawing, atom labeling and selected bond distances (in $\AA$ ) of relevant structures in the photodimerization path of $(\mathrm{AA})_{\text {syn-anti }}$ conformers of $(\mathrm{dA})_{\mathrm{n}}$ oligonucleotides: (a) $\phi-\mathrm{CI}^{*}$, corresponding to the conical intersection, (b) reaction intermediate $\phi \mathrm{I}$, (c) final photoproduct $\mathrm{AA}^{*}$ and (d) final photo-product $\mathrm{A}=\mathrm{A}$.

Excited state PCM/TD-M052X calculations, which have been successfully applied to the study of DNA photochemistry, ${ }^{9}, 14-15$ indicate that, for all conformers, the two lowest energy excited states in the Franck-Condon region correspond to symmetric/antisymmetric combinations (Frenkel excitons) of the lowest energy $\pi \pi^{*}$ 
excited state of adenine (Table SI-2), denoted as $\mathrm{L}_{\mathrm{a}}$. Geometry optimization of the $\mathrm{S}_{1}$ excited state leads to minima (Fig. 2 and Fig. SI-3) characterized by a close approach of the two bases. The $\mathrm{S}_{1}$ minimum found for (AA) $)_{\text {syn-anti }}$ exhibits very short ${ }^{5} \mathrm{C} 8-{ }^{3} \mathrm{C} 5$ and ${ }^{5} \mathrm{~N} 7-$ ${ }^{3} \mathrm{C} 6$ distances (the superscripts 5 and 3 correspond to the bases at the 5' and 3'-end, respectively). The $\mathrm{HOMO} \rightarrow \mathrm{LUMO}$ electronic transition involves transfer of an electron to an orbital with bonding character in respect to these atom pairs (Fig. 2). An extensive analysis of the potential energy surfaces along the ${ }^{5} \mathrm{C} 8-{ }^{3} \mathrm{C} 5$ coordinate shows that an extremely low energy barrier $(<0.08 \mathrm{eV})$ separates the $\mathrm{S}_{1}$ minimum from a crossing region with $S_{0}$ in the path leading to photodimerization.

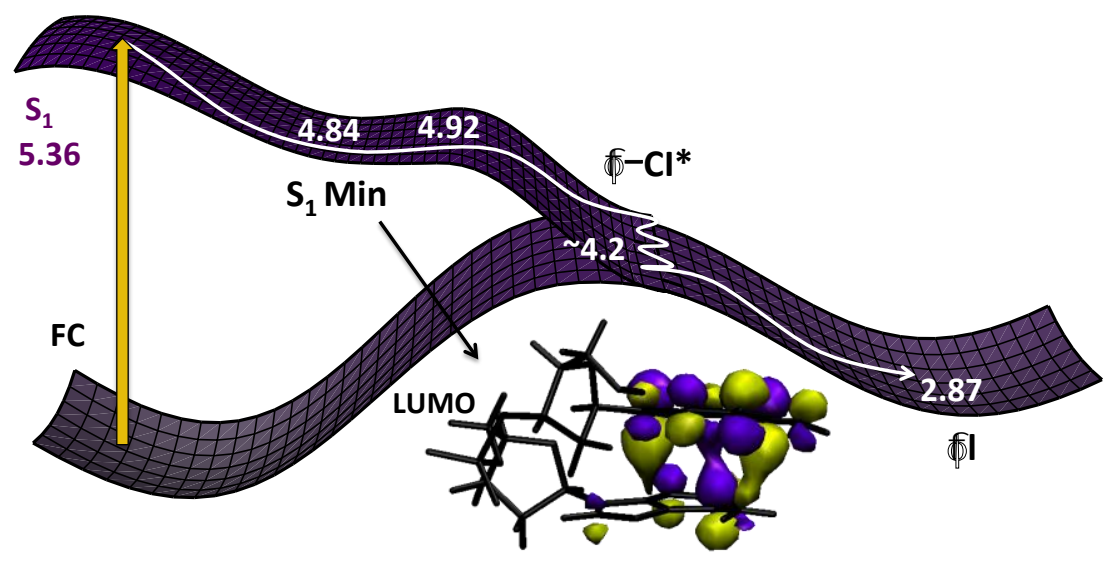

Figure 2. Schematic description of the potential energy surface in the path leading from the Franck-Condon state to the minimum of the $S_{1}$ excited state and, then, to the formation of the azetidine precursor $\phi I$. PCM/TD-M052X/6-31G(d) calculations for the in (AA) syn-anti conformer. The energy is given in $\mathrm{eV}$.

In general, TD-DFT cannot provide an accurate description of conical intersections However, in the case of several photodimerization processes in DNA, the description obtained for the $\mathrm{S}_{1} / \mathrm{S}_{0}$ crossing region by TD-M052X is very similar to that obtained by wavefunction based methods. ${ }^{9}$, $14-15$ A representative structure corresponding to this 
region $\left(\phi-\mathrm{CI}^{*}\right)$, is shown in Fig. 1a. Subsequent $\mathrm{S}_{1}$ geometry optimization leads to the photo-adduct $\phi \mathrm{I}$ presented in Fig. $1 \mathrm{~b}$.

According to our calculations, the three other conformers do not exhibit any significant tendency to photodimerization (see SI for details, Figs. SI-4, 5 and 6). The general picture obtained for (AA) anti-syn is qualitatively similar to that shown in Fig. 2 but the energy barrier separating the $S_{1}$ minimum from the crossing region is much higher $(\geq 0.33 \mathrm{eV})$. In the $\mathrm{S}_{1}$ minimum of $(\mathrm{AA})_{\text {syn-syn }}$ the ${ }^{5} \mathrm{C} 8$ and ${ }^{3} \mathrm{C} 8$ atoms of adenines are very close $(\sim 1.6 \AA)$; further approach leads to a crossing region with $S_{0}$ and decay back to the ground state of (AA) syn-syn. For (AA) anti-anti, the calculation along the ${ }^{5} \mathrm{C} 8-{ }^{3} \mathrm{C} 5$ coordinate involves a large increase in the energy $(>0.5 \mathrm{eV})$, making the access to $\phi \mathrm{I}$ impossible. Finally, in the case of (AA) anti-anti, we located another possible photo-adduct (see SI) where ${ }^{5} \mathrm{C} 5-{ }^{3} \mathrm{C} 5$ and ${ }^{5} \mathrm{C} 6$ ${ }^{3} \mathrm{C} 6$ are bonded. However, the latter photo-adduct is less stable than $\phi \mathrm{I}$ by $1.2 \mathrm{eV}$. Therefore, $\phi \mathrm{I}$ appears the only relevant intermediate in the path to $\mathrm{A}=\mathrm{A}$ and $\mathrm{AA}^{*}$ and corresponds to the species postulated 25 years ago by Sharma and Davies. ${ }^{5}$ 


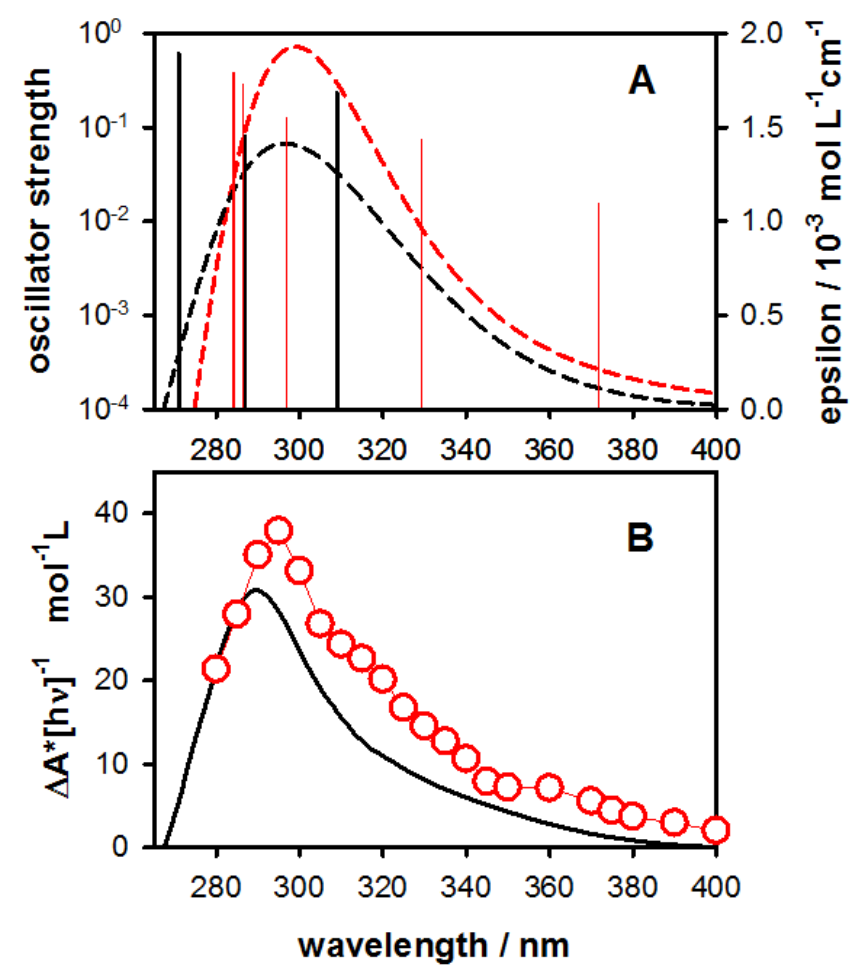

Figure 3. (A) Low energy electronic transitions of $\mathrm{A}=\mathrm{A}$ and $\mathrm{AA}^{*}$ dimers (black) and the precursor $\phi \mathrm{I}$ (red); PCM/TD-CAM-B3LYP/6-31+G(d,p)//PCM/M052X/6-31G(d) calculations. The energies have been homogenously decreased by $0.45 \mathrm{eV}$, which is the difference between the experimental absorption maximum of adenine and the vertical absorption energy computed at the adopted level of theory. In dashes: calculated differential absorption spectra corresponding to the disappearance of reacting adenines and the formation of $\phi \mathrm{I}$ (red) and $\mathrm{A}=\mathrm{A}$ and $\mathrm{AA}$ * (black, assuming that their yields are roughly equal, in line with literature $\left.{ }^{6}\right)$; the oscillator strength is proportional to the spectral area; the spectral width is $0.4 \mathrm{eV}$. (B) Spectrum obtained for $(\mathrm{dA})_{20}$ at $20 \mathrm{~ms}$ (red) compared to the difference of steady-state absorption spectra recorded after and before irradiation (black). The spectra are normalized by the concentration of absorbed photons $[\mathrm{hv}]$.

The computed absorption spectrum of $\phi \mathrm{I}$ (Fig. SI-7) exhibits two transitions located at energies lower than those of both the reacting adenines (AA) and the dimers $\mathrm{A}=\mathrm{A}$ and $\mathrm{AA}^{*}$ by at least 0.25 and $0.6 \mathrm{eV}$ (Fig. 3A). This finding suggests that, at wavelengths longer than $300 \mathrm{~nm}$, the $\phi \mathrm{I}$ spectrum is more intense compared to those of the reactants and the final photoproducts. On this ground, we searched the fingerprints of $\phi \mathrm{I}$ in the time-resolved spectra recorded by laser flash photolysis. This technique has been applied 
successfully to study the formation of 64PPs in thymine single strands from the oxetane intermediate. $^{16}$

The studied adenine eicosamers (dA) 20 (HPLC purified from Eurogentec Europe) were dissolved in phosphate buffer $\left(0.15 \mathrm{~mol} \cdot \mathrm{L}^{-1} \quad \mathrm{NaH}_{2} \mathrm{PO}_{4}, 0.15 \mathrm{~mol} \cdot \mathrm{L}^{-1} \mathrm{Na}_{2} \mathrm{HPO}_{4}\right)$. The solutions were excited at $266 \mathrm{~nm}$ with $5 \mathrm{~ns}$ pulses. We stress that such experiments are very delicate and specific experimental protocols, preventing excitation of UV-damaged oligomers are required (see SI). The average power used in our experiments varied from 0.5 to $2 \times 10^{6} \mathrm{~W} \cdot \mathrm{cm}^{-2}$. Under such experimental conditions, in addition to UV-induced dimerization, ionization processes give rise to adenine radicals. ${ }^{17}$ The typical absorption bands of the adenine radical are easily distinguishable in the $(\mathrm{dA})_{20}$ spectrum recorded at $1.7 \mu \mathrm{s}$. This is shown in Fig. 4A together with the spectrum of the deprotonated radical cation reported by Candeias and Steenken. ${ }^{18}$ The intensity of the band at $600 \mathrm{~nm}$ varies linearly with the concentration of ejected hydrated electrons $\left[\mathrm{e}^{-}\right]$(Fig. SI-8), determined from their absorption band peaking at $720 \mathrm{~nm} .{ }^{17,19}$ This is not the case for the UV band, whose shape changes with the intensity of the excitation pulse. When the ratio of ejected electrons versus absorbed photons $\left[\mathrm{e}^{-}\right] /[\mathrm{h} v]$ drops from $1.5 \times 10^{-3}$ to $0.3 \times 10^{-3}$, we can clearly distinguish a peak at $300 \mathrm{~nm}$ while the radical peak at $335 \mathrm{~nm}$ appears only as a shoulder (inset in Fig. 4A). 




Figure 4. Time-resolved absorption spectra (circles) obtained for $(\mathrm{dA})_{20}$ at $1.7 \mu \mathrm{s}(\mathbf{A})$ and $20 \mathrm{~ms}$ (B) following laser excitation at $266 \mathrm{~nm}$. The grey line in A corresponds to the spectrum of the adenine radical (from reference ${ }^{18}$; in arbitrary units). Inset in $\mathbf{A}$ : normalized spectra obtained for different ratios of ejected electron versus absorbed photons; $1.5 \times 10^{-3}$ (yellow) and $0.3 \times 10^{-3}$ (cyan). Inset in B: dependence of the transient absorbance at $340 \mathrm{~nm}$ and $20 \mathrm{~ms}$ on the concentration of absorbed photons.

At much longer times, when the adenine radicals have disappeared, we observe a large absorption band peaking at $300 \mathrm{~nm}$ with a weak tail extending to $420 \mathrm{~nm}$ (Fig. 4B). In contrast to the species issued from electron ejection, the intensity of the band at $20 \mathrm{~ms}$ scales with the concentration of absorbed photons (inset in Fig. 4B). The transient spectra obtained for aerated and Argon bubbled solutions are practically the same. These observations indicate that the chemical species responsible for the $300 \mathrm{~nm}$ band, which appears at times shorter than the time resolution of the experimental setup (50 ns; Fig. SI- 
9) originates from a singlet excited state. We have not observed any significant decay within $100 \mathrm{~ms}$. Beyond this time, the instability of the detector hinders the measurements. The spectrum at $20 \mathrm{~ms}$ is compared in Fig. 3B with the difference of the steady-state spectra obtained before and after $266 \mathrm{~nm}$ irradiation. The same differential spectrum is obtained using either a continuous light source or laser pulses of varying energy. In all cases, the spectrum intensity scales with the number of absorbed photons (Fig. SI-10) and it is not altered for several hours after irradiation; from these steady-state spectra and using the average molar absorption coefficient of the adenine dimers reported by Pörschke for $(\mathrm{dA})_{2},{ }^{1}$ we estimated that the dimerization quantum yield is $\sim 7 \times 10^{-3}$, which of the same order of magnitude as that reported by Clingen and Davies for poly(dA) $\left(3 \times 10^{-3}\right){ }^{6}$

The comparison of the two spectra in Fig. 3B shows that spectral changes occur between $100 \mathrm{~ms}$ and $5 \mathrm{~min}$, which is the time between irradiation and recording of the steady-state spectrum. The peak shifts from 300 to $295 \mathrm{~nm}$ and the intensity between 295 and $400 \mathrm{~nm}$ decreases significantly. These modifications compare nicely with the differences in the corresponding theoretical spectra (Fig. 3A), computed assuming that the two final photoproducts are formed with the same quantum yield.

In view of the above discussion, we assign the transient spectrum to the precursor $\phi I$. Its conversion to $\mathrm{A}=\mathrm{A}$ and $\mathrm{AA}^{*}$ is obviously slower compared with the evolution of oxetane to 64PPs in thymine strands, which takes place within $4 \mathrm{~ms}$, suggesting that the stability of the studied azetidine intermediate is larger than that ofthe oxetane. ${ }^{16}$ The stability of four-ring dimerization precursors has been correlated with the electronegativity of the involved heteroatoms. In the presence of sulphur atom, which is less electronegative than nitrogen and 
oxygen atoms, the reaction intermediate thietane is so stable that it has been isolated and characterized. ${ }^{20}$ Thus, it is not surprising that the Nitrogen containing precursor $\phi \mathrm{I}$, is less stable than thietane but longer lived than oxetane.

In conclusion, we studied adenine dimerization combining theoretical tools (molecular dynamics simulations and quantum mechanical methods) with steady-state and timeresolved absorption spectroscopy. We identified a reaction intermediate which is formed from a singlet excited state and characterized by a lifetime comprised between $0.1 \mathrm{~s}$ and a few minutes. We showed that the photodimerization reaction is governed by conformational equilibria.Different interbase stacking geometries lead to different patterns of bonding/non-bonding interactions between atoms of the two adenines, and, therefore, determinewhich photodimers can be potentially formed without a significant energy barrier. Moreover, some of the possible photoproducts do not correspond to stable minima, as shown by our results on $(\mathrm{AA})_{s y n-s y n}$, or they are less stable than the reactants. Consequently, adenine photodimerization is possible only for syn-anti conformers. This finding explains why base pairing,dramatically destabilizing syn conformers,diminishes drastically the quantum yield of the UV-induced adenine dimers. ${ }^{6}$ It would be interesting to perform the same type of investigation for other photodimerization reactions, not only inDNA strands but also in other multichromophoric systems whose geometry is characterized by many degrees of freedom.

Supporting Information:Computational Details, Additional Computational Results, Experimental Details, Additional Experimental Results, Cartesian Coordinates. 


\section{AUTHOR INFORMATION}

\section{Corresponding Authors}

dimitra.markovitsi@cea.fr

robimp@unina.it

The authors declare no competing financial interests.

\section{ACKNOWLEDGMENT}

Financial support from the French Agency for Research (ANR-10-BLAN-0809-01), the CNRSCNR PICS project ( $\left.{ }^{\circ} 6827-2015\right) /$ Bilateral CNR/CNRS and the Legge Regionale 5 Campania 2007 is acknowledged. 


\section{REFERENCES}

(1) Porschke, D.; Analysis of a Specific Photoreaction in Oligodeoxyadenylic and Polydeoxyadenylic Acids. J. Am. Chem. Soc. 1973,95, 8440-8446.

(2) Porschke, D.; Specific Photoreaction in Polydeoxyadenylic Acid. Proc. Natl. Ac. Sci. USA 1973,70, 2683-2686.

(3) Kumar, S.; Sharma, N. D.; Davies, R. J. H.; Phillipson, D. W.; McCloskey, J. A.; The Isolation and and Characterization of a New Type of Dimeric Adenine Photoproduct in UVIrradiated Deoxyadenylates. Nucl. Ac. Res. 1987,15, 1199-1216.

(4) Sharma, N. D.; Davies, R. J. H.; Extent of Formation of a Dimeric Adenine Photoproduct in Polynucleotides and DNA. J. Photochem. Photobiol. B-Biol. 1989,3, 247-258.

(5) Kumar, S.; Joshi, P. C.; Sharma, N. D.; Bose, S. N.; Davies, R. J. H.; Takeda, N.; McCloskey, J. A.; Adenine Photodimerization in Deoxyadenylate Sequences - Elucidation of the Mechanism through Structural Studies of a Major D(ApA) Photoproduct. Nucl. Ac. Res. 1991,19, $2841-2847$.

(6) Clingen, P. H.; Davies, R. J. H.; Quantum Yields of Adenine Photodimerization in Poly(Deoxyadenylic Acid) and DNA. J. Photochem. Photobiol. B-Biol. 1997,38, 81-87.

(7) Wang, Y. S.; Taylor, J. S.; Gross, M. L.; Isolation and Mass Spectrometric Characterization of Dimeric Adenine Photoproducts in Oligodeoxynucleotides. Chem. Res. Toxicol. 2001,14, 738-745.

(8) Wang, Y. S.; Taylor, J. S.; Gross, M. L.; Fragmentation of Photomodified Oligodeoxynucleotides Adducted with Metal Ions in an Electrospray-Ionization Ion-Trap Mass Spectrometer. J. Am. Soc. Mass Spectr. 2001,12, 1174-1179. 
(9) Banyasz, A.; Douki, T.; Improta, R.; Gustavsson, T.; Onidas, D.; Vayá, I.; Perron, M.; Markovitsi, D.; Electronic Excited States Responsible for Dimer Formation Upon UV Absorption Directly by Thymine Strands: Joint Experimental and Theoretical Study. J. Am. Chem. Soc. 2012,134, 14834-14845.

(10) Cadet, J.; Mouret, S.; Ravanat, J.-L.; Douki, T.; Photoinduced Damage to Cellular DNA: Direct and Photosensitized Reactions. Photochem. Photobiol. 2012,88, 1048-1065.

(11) Richa; Sinha, R. P.; Haeder, D.-P.; Physiological Aspects of UV-Excitation of DNA. Top. Curr. Chem.2015;356, 203-248.

(12) Zhao, Y.; Truhlar, D. G.; Density Functionals with Broad Applicability in Chemistry. Acc. Chem. Res. 2008,41, 157-167.

(13) Tomasi, J.; Mennucci, B.; Cammi, R.; Quantum Mechanical Continuum Solvation Models. Chem. Rev. 2005,105, 2999-3093.

(14) Esposito, L.; Banyasz, A.; Douki, T.; Perron, M.; Markovitsi, D.; Improta, R.; Effect of C5-Methylation of Cytosine on the Photoreactivity of DNA: A Joint Experimental and Computational Study of TCG Trinucleotides. J. Am. Chem. Soc. 2014,136, 10838-10841.

(15) Improta, R.; Photophysics and Photochemistry of Thymine Deoxy-Dinucleotide in Water: A Pcm/Td-Dft Quantum Mechanical Study. J. Phys. Chem. B 2012,116, 14261-14274.

(16) Marguet, S.; Markovitsi, D.; Time-Resolved Study of Thymine Dimer Formation. J. Am. Chem. Soc. 2005,127, 5780-5781.

(17) Marguet, S.; Markovitsi, D.; Talbot, F.; One and Two Photon Ionization of DNA Single and Double Helices Studied by Laser Flash Photolysis at 266 Nm. J. Phys. Chem. B 2006,110, 11037-11039. 
(18) Candeias, L. P.; Steenken, S.; Ionization of Purine Nucleosides and Nucleotides and Their Components by 193-nm Laser Photolysis in Aqueous Solution: Model Studies for Oxidative Damage of DNA. J. Am. Chem. Soc. 1992,114, 699-704.

(19) Buxton, G. V.; Greenstock, C. L.; Helman, W. P.; Ross, A. B.; Critical Review of Rate Constants for Reactions of Hydrated Electrons, Hydrogen Atoms and Hydroxyl Radicals (.OH/O.-) in Aqueous Solution. J. Phys.Chem. Ref. Data 1988,17, 513-886.

(20) Clivio, P.; Fourrey, J.-L.; Gasche, J.; DNA Photodamage Mechanistic Studies: Characterization of a Thietane Intermediate in a Model Reaction Relevant to "6-4 Lesions". $J$. Am. Chem. Soc. 1991,113, 5481-5483. 